\title{
Versatile biomimetic conductive polypyrrole films doped with hyaluronic acid of different molecular weights
}

Semin Kim ${ }^{a} \neq$, Yohan Jang ${ }^{a} \neq$, Minsu Jang ${ }^{a}$, Ahyoun Lim ${ }^{b}$, John G. Hardy ${ }^{c}$, Hyun S. Park ${ }^{b}$, and Jae Young Lee ${ }^{a, d^{*}}$

${ }^{\text {a }}$ School of Materials Science and Engineering, Gwangju Institute of Science and Technology, Gwangju 61005, Republic of Korea

${ }^{\mathrm{b}}$ Korea Institute of Science and Technology, Fuel Cell Research Center, Hwarangro 14-gil 5, Seoul 02792, Republic of Korea

${ }^{\mathrm{c}}$ Department of Chemistry and Materials Science Institute, Lancaster University, Lancaster, Lancashire, LA1 4YB, United Kingdom

\footnotetext{
${ }^{\mathrm{d}}$ Department of Biomedical Science and Engineering, Gwangju Institute of Science and Technology, Gwangju 61005, Republic of Korea
}

*E-mail: jaeyounglee@gist.ac.kr (J. Y. Lee)

\$ These authors contributed equally to this work.

KEYWORDS: conductive polymer; hyaluronic acid; molecular weight; cellular interactions; bioelectrodes 


\begin{abstract}
Abbreviations
PPy, polypyrrole; HA, hyaluronic acid; PPy/HA, Hyaluronic acid-doped polypyrrole; MW, molecular weight; CPs, Conductive polymers; PEDOT, Poly(3,4-ethylenediowythiophene); ECM, extracellular matrix; HUVECs, human umbilical cord endothelial cells; ITO, indiumtin-oxide; QCM, quartz crystal microbalance; CV, cyclic voltammetry; XPS, X-ray photoelectron spectroscopy; AFM, Atomic force microscopy; KFPM, Kelvin force probe mode; TBO, Toluidine Blue O; WCA, Water contact angle; EMG, electromyography.
\end{abstract}

\begin{abstract}
Electrically conductive polypyrrole (PPy) is an intriguing biomaterial capable of efficient electrical interactions with biological systems. Especially, biomimetic PPy-based biomaterials incorporating biomolecules such as hyaluronic acid (HA), can impart the characteristic biological interactions with living cells/tissues to the conductive biomaterials. Here we report the effects of the molecular weight (MW) of HA on PPy-based biomaterials. We utilized HA of a wide range of MW $\left(35 \times 10^{3} \mathrm{Da}-3 \times 10^{6} \mathrm{Da}\right)$ as dopants during the electrochemical production of PPy/HA films and their characterization of materials and cellular interactions. With increases in the MWs of HA dopants, PPy/HA exhibited more hydrophilic, higher electrochemical activity and lower impedance. In vitro studies revealed that PPy films doped with low MW HA were supportive to cell adhesion and growth, while PPy films doped with high MW HA were resistant to cell attachment. Subcutaneous implantation of the PPy/HA films for 4 weeks revealed that all the PPy/HA films were tissue compatible. We successfully demonstrate the importance of HA dopant MWs in modulating the chemical and electrical properties of the materials and cellular responses to the materials. Such materials have potential for various biomedical applications, including as tissue scaffolds for tissue engineering scaffolds and as electrodes for neural recording and neuromodulation.
\end{abstract}




\section{Introduction}

Electrically conductive biomaterials have received great attention because of their potential to efficiently mediate electrical signals between electrodes and biological systems.[1-3] Conductive polymers (CPs) such as polyaniline, polypyrrole and PEDOT, are an interesting class of materials to use in the development of electrically conductive biomaterials because of their good conductivity, ease of synthesis and biocompatibility.[4-6] These interesting properties make CP-based biomaterials attractive for various biomedical applications, such as bioelectrodes, prosthetic probes and tissue scaffolds.[7-12] CPs have been engineered to present various characteristics, such as topographical cell guidance, mechanical flexibility, and biological activities,[13-17] enabling the characteristics of the CP-based biomaterials to be tailored for their specific applications. For example, CPs able to support cell growth and encourage specific maturation are potentially useful for tissue engineering;[18-20] whereas, non-fouling and/or resistance to non-specific protein/cell adhesion are useful for the development of highly sensitive and stable bioelectrodes.[21,22] Therefore, the design and fabrication of specific functional CPs is pivotal to achieve their intended performance.

Biomimetic and biologically active CPs have been fabricated by incorporating extracellular matrix (ECM) components (such as nucleotides, proteins, and polysaccharides) into the materials to generate biocompatible and interactive materials.[23-25] Polypyrrole (PPy) is one of the CPs that has been extensively studied as a biomaterial. CPs, including PPy, inherently require dopants during polymerization to ensure the charge on their backbones is neutralized (in the case of PPy, the backbone is positively charged and therefore the dopants are negatively charged).[26] The choice of dopant critically influences the mechanical, electrical and biological properties of the resulting CP-based biomaterials.[27-29] Hyaluronic acid (HA) is a major component of connective tissues in the body and is widely used for the production of biocompatible materials. Importantly, HA is an anionic polysaccharide 
consisting of D-glucuronic acid and N-acetyl-D-glucosamine repeating units.[30] For the production of biomimetic CPs, HA-doped PPy (PPy/HA) was previously prepared and its characteristics, including cellular interactions, were examined. Schmidt and colleagues electrochemically synthesized HA-doped PPy (PPy/HA) films and found that they could promote vascularization.[31] The Wallace group examined the electromechanical and biological characteristics of PPy/HA for biological applications, demonstrating that PPy/HA supported cell growth.[32] However, to the best of our knowledge, there has not been a systematic study of the role that the MW of HA may play in the properties of the materials, despite the fact that the properties of HA-based materials (e.g. viscosity, hydrophilicity, degradation rate, and cell receptor signaling) are dependent on its molecular weight (MW).[33-36] For example, Li et al. reported that high MW HA $\left(5 \times 10^{5} \mathrm{Da}\right)$ coating on amine-rich dopamine-hexamethyldiamine surfaces suppressed the adhesion and proliferation of HUVECs (human umbilical cord endothelial cells), while coating low MW HA $\left(4 \times 10^{3}\right.$ Da) led to good adhesion and proliferation of the HUVECs.[37]

The focus of this study was to systematically explore the characteristics of PPy/HA prepared with HA of various MWs (ranging from $35 \times 10^{3} \sim 3.0 \times 10^{6} \mathrm{Da}$ ) (Scheme 1), and the molecular weight of HA in vivo is typically between $5 \times 10^{3}$ and $20 \times 10^{6} \mathrm{Da}$.[38,39] The material properties and in vitro cellular interactions using SH-SY5Y neuroblastoma and NIH3T3 fibroblasts and in vivo tissue compatibility were studied. EMG signals were also recorded with the PPy/HA-modified electrodes in vivo, validating the function of these biomaterials in vivo. 

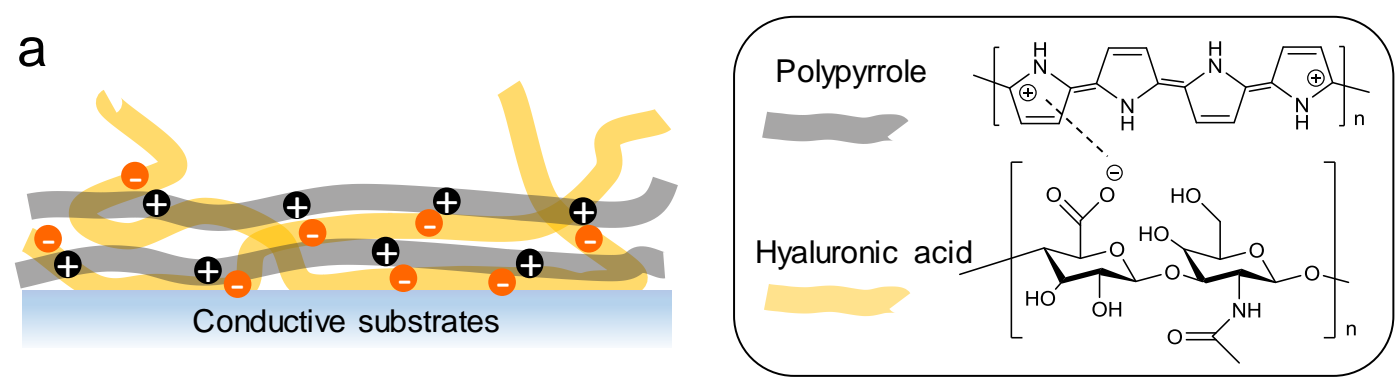

b

Effects of molecular weight



High MW

- Increase hydrophilicity

- Decreased cell adhesion

- Increased conductivity
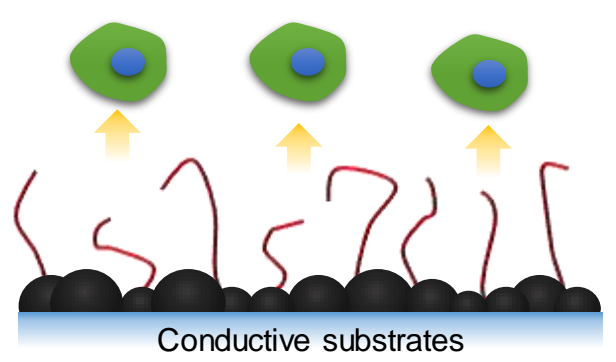

Scheme 1. Schematic of hyaluronic acid-doped PPy (PPy/HA). (a) Structures of PPy and HA in PPy/HA. (b) Summarized properties of PPy/HA prepared with different molecular weight HA.

\section{Materials and Methods}

\subsection{Materials}

Pyrrole, potassium ferrocyanide $\left(\mathrm{K}_{4} \mathrm{Fe}(\mathrm{CN})_{6}\right)$, potassium ferricyanide $\left(\mathrm{K}_{3} \mathrm{Fe}(\mathrm{CN})_{6}\right)$, potassium chloride, acetic acid, sodium hydroxide and aluminum oxide were purchased from SigmaAldrich (St. Louis, MO, USA). HA $\left(35 \times 10^{3} \mathrm{Da}\right)$ was obtained from Baoding Faithful Industry Co., Ltd. (China), HA $\left(100 \times 10^{3} \mathrm{Da}\right.$ and $\left.500 \times 10^{3} \mathrm{Da}\right)$ was purchased from Lifecore Co. (Chaska, MN, USA). HA $\left(1 \times 10^{6}\right.$ Da and $\left.3 \times 10^{6} \mathrm{Da}\right)$ was obtained from LG Chem (Daejeon, Republic of Korea). Dulbecco's modified Eagle's medium (DMEM) and fetal bovine serum (FBS) were obtained from Hyclone (Logan, UT, USA). Dulbecco's 
phosphate buffered saline (DPBS). Penicillin streptomycin and trypsin/EDTA were purchased from Gibco (Grand Island, NY, USA). WST solution was obtained from EZ-Cytox (DoGen, Seoul, Republic of Korea). Aelxa-fluor 488-conjugated phalloidin and DAPI were purchased from Invitrogen (Carlsbad, CA, USA).

\subsection{Electrochemical synthesis of PPy/HA films}

PPy/HA films were electrochemically synthesized on indium-tin-oxide coated glass electrodes (ITO; AMG, Republic of Korea). ITO slides were cleaned by sequential sonication in acetone, methanol, and deionized water (DIW) for 10 min respectively. Pyrrole was purified by passing it through alumina column. Individual polymerization solution containing $0.1 \mathrm{M}$ pyrrole and $2 \mathrm{mg} / \mathrm{mL} \mathrm{HA}\left(35 \times 10^{3} \mathrm{Da}, 100 \times 10^{3} \mathrm{Da}, 500 \times 10^{3} \mathrm{Da}, 1 \times 10^{6} \mathrm{Da}\right.$, and $3 \times 10^{6}$ Da) were prepared and sonicated for $360 \mathrm{~s}$ in the bath sonicator (B5510, Branson) prior to electrochemical polymerization. Electrochemical deposition was performed using a potentiostat (VersaSTAT3 electrochemical working station, Princeton Applied Research) with a three-electrode setup. A platinum wire and standard calomel electrode (SCE) were used as counter electrode and reference electrode, respectively. PPy/HA was polymerized by applying a constant potential $(0.8 \mathrm{~V}$ v.s. SCE) for $10 \mathrm{~min}$. The electrodes were then washed with DIW and dried in air. A quartz crystal microbalance (QCM; QCM922A, SEIKO EG\&G, Japan) was used to monitor the PPy/HA deposition during the electrochemical polymerization of PPy/HA. For the electrochemical impedance spectroscopy (EIS) and cyclic voltammetry (CV), ITO electrode was covered with a tape film that had a hole in the middle $\left(0.283 \mathrm{~cm}^{2}\right)$ to ensure the electrodes possessed the same surface area.

\subsection{Electrochemical characterization of the PPy/HA films}

EIS measurement with bare or PPy-HA modified electrodes was performed in PBS containing $5 \mathrm{mM}$ potassium $\mathrm{K}_{3} \mathrm{Fe}(\mathrm{CN})_{6}$ (Sigma Aldrich). An alternative sinusoidal potential of $10 \mathrm{mV}$ 
and a DC potential of $0 \mathrm{~V}$ (vs. open circuit voltage) was applied in a range of $10^{-1}-10^{5} \mathrm{~Hz}$. The obtained data was further analyzed to calculate the circuit parameters using Zsimpwin software. Cyclic voltammery $(\mathrm{CV})$ was performed in $0.1 \mathrm{M} \mathrm{KCl}$ solution containing $5 \mathrm{mM}$ $\mathrm{K}_{3} \mathrm{Fe}(\mathrm{CN})_{6}$. Voltages were scanned from $-0.4 \mathrm{~V}$ to $0.6 \mathrm{~V}$ at a scan rate of $10 \mathrm{mV} / \mathrm{s}$. X-ray photoelectron spectroscopy (XPS) was performed for surface elemental analysis by using VG Multilab 2000 spectrometer (Thermo VG Scientific) under ultra-high vacuum. The survey scan data were obtained with a pass energy of $200 \mathrm{eV}$. High-resolution spectra of $\mathrm{C}_{1 \mathrm{~s}}$ scan were deconvoluted using XPSPEAK software. Atomic force microscopy (AFM, XE-100, PARK System, Republic of Korea) was operated in a non-contact mode with NSC14-Cr/Au cantilever. Scans of $2 \mu \mathrm{m} \times 2 \mu \mathrm{m}$ area were acquired at a scan rate of $0.9 \mathrm{~Hz}$. Surface roughness of the samples was calculated from the data by XEI Software 4.3 (Park Systems, Republic of Korea). The surface potential distribution of the PPy/HA samples was measured using XE-100 in Kelvin force probe mode (KFPM) in air. Resonance frequency of $70 \mathrm{kHz}$ was employed for imaging at a scan rate of $0.9 \mathrm{~Hz}$. The topography of the sample area $(2 \mu \mathrm{m}$ $\times 2 \mu \mathrm{m}$ ) was acquired in AC mode while recording the surface potentials of the PPy/HA films. Relative differences in the surface potentials across the films were calculated by using XEI Software (Park Systems, Suwon, Republic of Korea). Toluidine Blue O (TBO) assay was performed to quantify the surface concentration of free carboxyl groups according to the previously described method.[40] In brief, samples were immersed in an aqueous TBO solution $(0.5 \mathrm{mM}, \mathrm{pH} 10)$ for $6 \mathrm{~h}$ in room temperature. Then, the samples were carefully washed with sodium hydroxide solution $(10 \mathrm{mM})$ to remove the unbound TBO. TBO dye was detached by incubating the samples in acetic acid solution (0.082 $\mathrm{M}, \mathrm{pH} 2)$ for $20 \mathrm{~min}$. Absorbance of the solution was measured by using an ultraviolet-visible spectroscopy (UVvis, Biodrop Duo, Biodrop, UK) to quantify the amount of the TBO bound with the samples. Water contact angle (WCA) was measured with a goniometer (Phoenix 300, SEO Co., AZ, 
USA) to study the wettability of the samples. A drop $(2 \mu \mathrm{L})$ of DIW was dropped on the sample at room temperature.

\subsection{In vitro cell culture studies}

Mouse NIH 3T3 fibroblasts and human SH-SY5Y neuroblastoma were maintained on a tissue culture plate (Nunc, Roskilde, Denmark) in a $5 \% \mathrm{CO}_{2}$ incubator at $37^{\circ} \mathrm{C}$ in a culture medium consisting of DMEM, 10\% heat-inactivated FBS, and 1\% penicillin/streptomycin solution. Prior to cell seeding, the substrates were sterilized by exposure to UV for $2 \mathrm{~h}$. SHSY5Y cells were seeded on the sample at a density of 15,000 cells per $\mathrm{cm}^{2}$ and incubated in a humidified incubator with $5 \% \mathrm{CO}_{2}$ at $37^{\circ} \mathrm{C}$. NIH3T3 cells were seeded at a density of 8,000 cells per $\mathrm{cm}^{2}$ and incubated in a humidified incubator with $5 \% \mathrm{CO}_{2}$ at $37^{\circ} \mathrm{C}$ for 7 days. After incubation, the samples were fixed in 5\% glutaraldehyde at room temperature for $2 \mathrm{~h}$. The samples were then washed with DPBS. Next, the samples were incubated in permeabilizing/blocking solution $(0.1 \%$ Triton X100, 2\% BSA, in DPBS) at room temperature for $10 \mathrm{~min}$ and then washed with DPBS. The samples were incubated in Alexafluor 488-conjugated phalloidin (1:200 in blocking solution) at room temperature for $20 \mathrm{~min}$, followed by two times washes with DPBS. The samples were then incubated with 4'-6diamidino-2-phenylindole (DAPI) solution (1:2500 in DPBS) at room temperature for $3 \mathrm{~min}$ and washed three times with DPBS. Fluorescence images were acquired using a fluorescence microscope (DMI3000B, Leica, Germany).

Metabolic activity of cells growing on various PPy/HA substrates was measured by WST assay. For the metabolic activity measurement, 3T3 cells and SH-SY5Y cells were seeded at densities of 4,000 cells and 10,000 cells per well, respectively, and cultured up to for 7 days. For quantification, WST solution $(100 \mu \mathrm{L})$ was added to individual sample wells. After $2 \mathrm{~h}$ of incubation, solution was transferred to 96 well plates and its absorbance at $450 \mathrm{~nm}$ was measured using a microplate reader (SpectraMax M2, CA, USA). 


\subsection{In vivo tissue compatibility}

All animal experiments were performed in accordance with permission from a committee on animal research and ethics in Gwangju Institute of Science and Technology (GIST), Republic of Korea (Approval number: GIST-2017-044). In vivo tissue compatibility was conducted by subcutaneous implantation of PPy/HA films to male BALB/c mice (six weeks old). Prior to implantation, the PPy/HA-modified electrodes were first immersed in $70 \%$ ethanol for sterilization for $1 \mathrm{~h}$, followed by thorough washing with sterile DPBS. The mice were anesthetized with 2\% isoflurane (Ankuk Inc., Republic of Korea). Shaved skin was disinfected with $70 \%(\mathrm{v} / \mathrm{v}$ ) ethanol, and the skin at the back of the animal was incised and a subcutaneous pocket was secured to the left and right of the incision. Two electrodes were implanted in each pocket, and the skin was sutured using a silk thread. Three mice were used for each experimental condition - bare, PPy/HA $\mathrm{H}_{35 \mathrm{k}}, \mathrm{PPy} / \mathrm{HA}_{100 \mathrm{k}}, \mathrm{PPy} / \mathrm{HA}_{500 \mathrm{k}}, \mathrm{PPy} / \mathrm{HA}_{1 \mathrm{M}}$, and $\mathrm{PPy} / \mathrm{HA}_{3 \mathrm{M}}$. Four weeks later the mice were sacrificed. Skin and muscle tissues implanted with electrodes were fixed in $3.7 \%$ formaldehyde for $3 \mathrm{~d}$ and then embedded in paraffin. Paraffinembedded tissue blocks were sectioned using a microtome to yield slides with a thickness of 5 $\mu \mathrm{m}$. For histological analyses, Masson Trichrome staining was performed according to the manufacturer's protocol. Images of the stained tissues were acquired using an optical microscope (Leica, Germany).

\subsection{In vivo EMG signal measurement}

Adult female rats (Orient Bio Inc., Republic of Korea) weighing approximately $350 \mathrm{~g}$ were used to measure EMG signal. Rats were anesthetized with 3\% isoflurane. Rats were placed on a heating pad $\left(37^{\circ} \mathrm{C}\right)$ to maintain body temperature and anesthetized with $3 \%$ isoflurane solution (Ankuk Inc., Republic of Korea). Concentric needle electrodes (Chalgren Enterprises, Inc, Gilroy, CA, USA) were electrochemically modified with $\mathrm{PPy} / \mathrm{HA}_{35 \mathrm{k}}$ and $\mathrm{PPy} / \mathrm{HA}_{3 \mathrm{M}}$ as 
described above. The PPy/HA-modified and bare electrodes were immersed in $70 \%$ ethanol for sterilization for $1 \mathrm{~h}$, followed by thorough washing with sterile DPBS. These electrodes were inserted into rat tibialis anterior. To obtain the EMG signal, a Plexigals-platinum electrode was used to stimulate the sciatic nerve with a voltage of $0.7 \mathrm{~V}$. EMG signals were recorded from the various needle electrodes using a Biopac MP36 instrument (Biopac Systems, Inc., CA, USA).

\subsection{Statistical evaluation}

All tests were performed at least in triplicate and results were reported as the mean \pm standard deviation (SD) unless otherwise noted. Results between groups were analyzed using one-way ANOVA, with appropriate corrections such as Tukey's post hoc tests used for comparisons. Statistical analyses were performed using Origin software. Differences were considered significant at $p<0.05$. For in vitro cell culture studies, homogeneity of variance was checked with Levene's test and the statistical signficance was evaluated using two-way ANOVA with Bonferroni's post-test.

\section{Results and discussion}

\subsection{Electrochemical polymerization of PPy/HA}

HA of five different MWs $\left(35 \times 10^{3}, 100 \times 10^{3}, 200 \times 10^{3}, 1 \times 10^{6}\right.$ and $\left.3 \times 10^{6} \mathrm{Da}\right)$ were used as dopants for the electrochemical synthesis of various PPy/HA to study the effects of the MWs of HA dopants on PPy material characteristics. The MW of HA in vivo was reported to be between $5 \times 10^{3}$ and $20 \times 10^{6} \mathrm{Da}$.[39] Therefore we selected the HA with MWs in this range to prepare biomimetic PPy/HA materials. HA-doped PPy was electrochemically deposited at a constant potential $0.8 \mathrm{~V}$ (vs SCE) for $10 \mathrm{~min}$. The samples were named PPy/HA $x$, in which $x$ indicates the MW (Da) of the respective HA dopant. During the electrochemical polymerization of PPy/HA, current, transferred charges and deposited masses 
were monitored (Figure 1). For all PPy/HA samples, the current gradually increased during electrochemical deposition which is likely to be because the increases in the surface areas of the electrodes. Interestingly, the deposition charge for $\mathrm{PPy} / \mathrm{HA}_{35 \mathrm{k}}$ was higher than other PPy/HA films. This observation is likely to be because HA with MWs greater than $35 \mathrm{kDa}$ diffuse more slowly to the vicinity of the polymerizing electrode. Quartz crystal microbalance (QCM) measurements during electrochemical deposition were employed to monitor the deposition of PPy/HA in a real-time manner. An overall relationship between the MWs of HA dopants and the deposited mass appeared to be a ' $U$ ' shape. This interesting phenomenon can be accounted for by two possible reasons: 1) as the MW of HA increases, the solution viscosity greatly increases and can hinder diffusion of pyrrole monomers and dopants toward the electrode surfaces for deposition, and 2) polyanionic HA dopants can facilitate PPy polymerization by providing extra negative charges to the positively charged PPy chains growing from the surface of the electrode (i.e. the anionic charge on large HA strands might be more than required for neutralization of the positively charged PPy backbone); thus, HA chains on the growing PPy/HA surfaces provide negatively charged templates that can induce both polymerization and deposition of growing PPy/HA. Furthermore, analysis of film thicknesses showed a similar trend with QCM and electrodeposition (Figure 1e). 
a



b

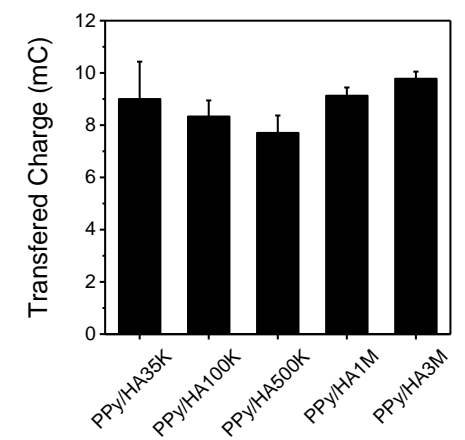

C

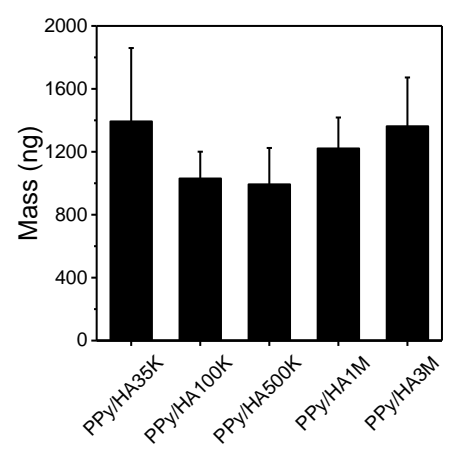

d

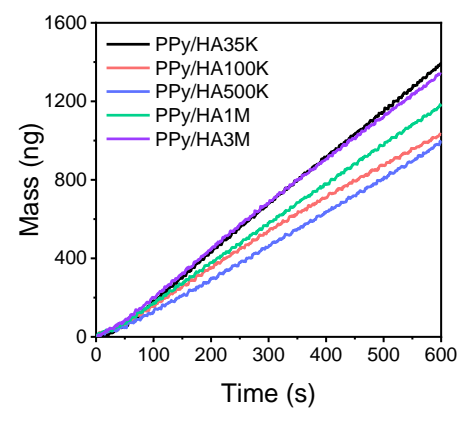

e



Figure 1. The electrochemical polymerization of PPy/HA on ITO electrodes using HA of different MW. (a) Currents during the electrochemical polymerization of $\mathrm{PPy} / \mathrm{HA}$ at a constant voltage $(0.8 \mathrm{~V}$ vs $\mathrm{SCE})$. (b) Transferred charges during the electrochemical polymerization. (c) Mass change profiles of the electrodes and (d) deposited mass for $600 \mathrm{~s}$. (e) Thickness of PPY/HA films. Thickness of each sample was measured by profilometry. $n=3$. Asteriks indicate statistical significance $(p<0.05)$.

\subsection{Surface characterization of PPy/HA coated ITO}

The hydrophilicity of the PPy/HA films was compared by measuring water contact angles (WCA) (Figure 2a, b). Overall, HA-doped PPy films exhibited hydrophilic surfaces (19.4$\left.40.6^{\circ}\right)$, which is a dramatic decrease from the bare ITO surface $\left(74.2^{\circ}\right)$. As the MWs of HA increased, the WCAs of PPy/HA gradually decreased. PPy films doped with the highest MW HA, i.e., PPy/HA3M, showed the lowest water contact angle $\left(\sim 19.4^{\circ}\right)$. This is likely to be because the backbone of higher MW HAs remains exposed on the surface of the electrodes with more dynamic chain movement, which results in charged surfaces and increased 
hydrophilicity. The hydrophilicity of biomaterials affects a broad range of interactions with biological systems including biomolecule adsorption and cell attachment. A hydrophilic surface forms a hydration layer that can exhibit antifouling properties resisting adsorption of proteins and cells.[41-43] To study the surface charge of the electrodes, a Toluidine blue $\mathrm{O}$ assay (TBO) was performed to quantify the free carboxylic groups in HA on PPy/HA surfaces (Figure 2c). Amounts of free carboxylic groups, indicative of free HA, on the surfaces greatly increased as the MWs of HA dopant in PPy/HA increased. For example, PPy/HA500k, PPy/HA1M and PPy/HA3M had free carboxylic groups 1.5, 3.5, and 5.2 times more than PPy/HA35k, respectively. These results suggested that the electrochemical PPy polymerization with high MW HA dopants result in surfaces displaying portions of HA chains not solely present to dope the PPy. Furthermore, X-ray photoelectron spectroscopy (XPS) was employed to analyze the chemical composition of PPy/HA film surfaces (Figure 2d, e, f). A peak intensity of C-C/C-H $(284.8 \mathrm{eV})$ was low for the PPy/HA of low MW HA. Peak intensities of oxygenated carbons (i.e., $\mathrm{C}-\mathrm{O}$ at $286.3 \mathrm{eV}$ and $\mathrm{C}=\mathrm{O}$ at $287.9 \mathrm{eV}$ ) were higher for the PPy/HA of higher MW HA dopants. Larger portions of $\mathrm{C}-\mathrm{O}$ and $\mathrm{C}=\mathrm{O}$ carbons on the PPy/HA of high MW HA confirm the presence of HA chains not solely present to dope the PPy. The surface atomic compositions also exhibited higher carbon-to-nitrogen $(\mathrm{C} / \mathrm{N})$ atomic ratios from the PPy/HA of higher MW HA (Figure 2e). WCA measurement, surface carboxylic acid quantification, and XPS confirm the components of the HA chains present on the surface of the PPy/HA coated electrodes that are not solely involved in doping the PPy, particularly when PPy/HA was synthesized with high MW HA. The "free" parts of the HA chains on PPy/HA films may play important roles in the surface morphologies, charges and electron transfers, and interactions with biological systems. 
a

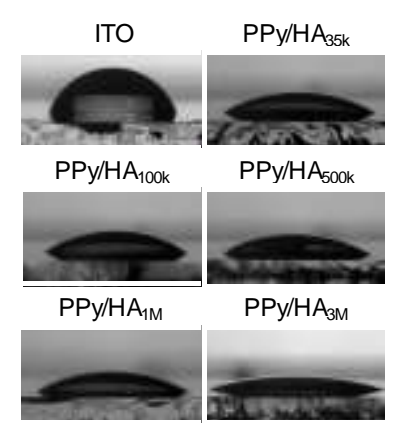

d

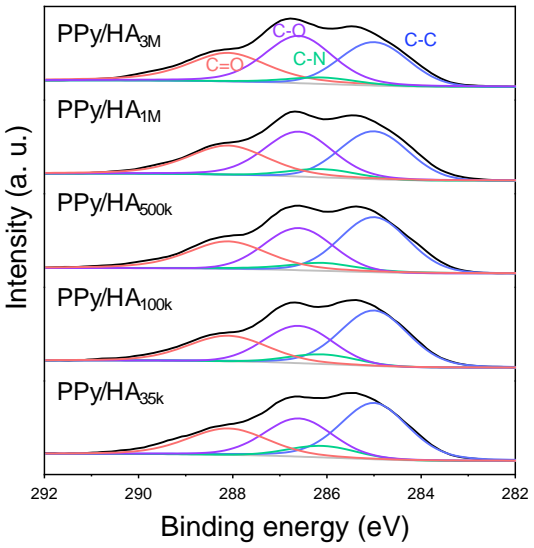

b



e

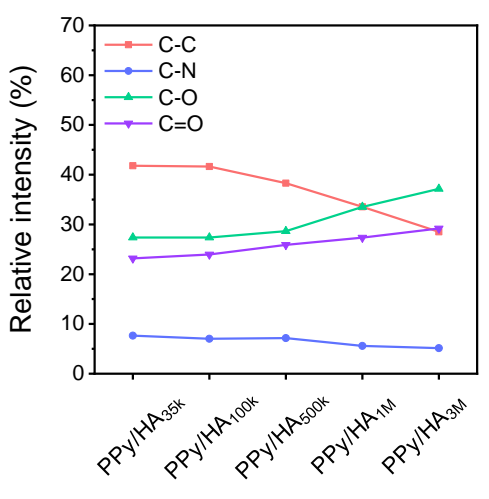

C

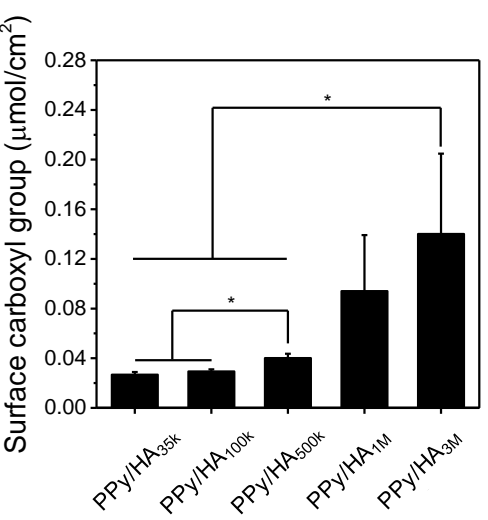

f

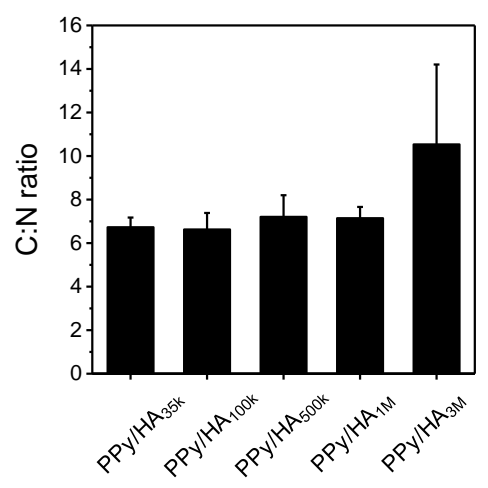

Figure 2. Surface characterization of the various PPy/HA substrates. (a) Images and (b) A bar graph of water contact angles. (c) Surface carboxylic groups on the samples. (d) High resolution of C1s spectra. (e) Relative intensities of various carbon peaks from the $\mathrm{C}_{1 \mathrm{~s}}$ spectra (d). (f) Carbon-to-nitrogen atomic ratios $(\mathrm{C} / \mathrm{N})$ of the PPy/HA surfaces.

Surface topographies and surface potentials of various PPy/HA films were analyzed using atomic force microscope (AFM) and Kelvin probe force microscope (KPFM). PPy/HA samples except PPy/HA $3 \mathrm{M}$ showed nodular features over the surfaces (Figure 3a). Nodular structures were typical for PPy doped with polysaccharides, such as HA and heparin. Nodule sizes were not significantly different among the samples (Supplementary Information Figure S1). In addition, the surface roughness appeared to become smoother as the dopant MW increased; however, the difference was not significant except PPy/HA $35 \mathrm{k}$. For example, PPy/HA $35 \mathrm{k}$ and PPy/HA3M had surface roughness of $3.87 \mathrm{~nm}$ and $3.21 \mathrm{~nm}$, respectively 
(Figure 3b). HA larger than $35 \times 10^{3}$ Da might aid organized polymerization and deposition by providing anionic character on the electrode surfaces. Additionally, KPFM was employed to study the surface potential and its distribution on PPy/HA surfaces (Figure 3c, Supplementary Information Figure S2). Surface potentials were similar and uniform for all the samples. R.M.S. surface potentials were approximately 3.7-3.8 mV for all PPy/HA samples.
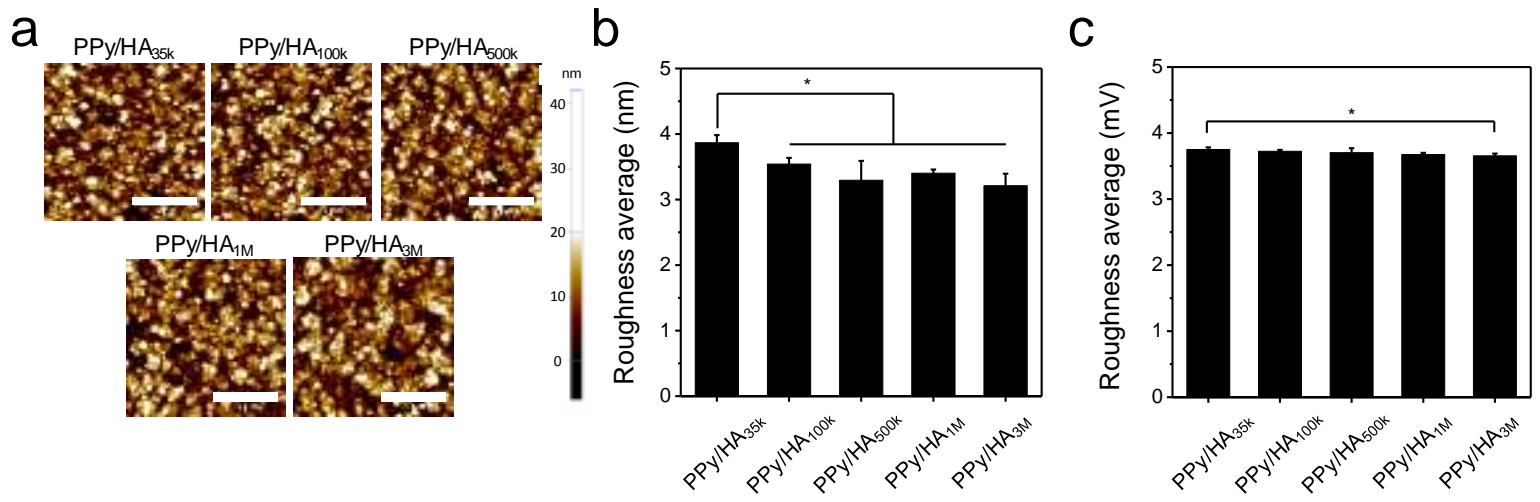

Figure 3. (a) AFM images and (b) surface roughness of various PPy/HA samples. Scale bars represent $2 \mu \mathrm{m}$. (c) Average deviations of surface potentials of various PPy/HA samples measured by KPFM.

\subsection{Electrochemical properties of PPy/HA film}

Electrical/electrochemical properties of conductive biomaterials are critical for their performances for use as bioelectrodes, biosensors, and tissue engineering scaffolds to efficiently mediate electrical signals. Electrochemical impedance spectroscopy (EIS) of PPy/HA-modified electrodes was conducted in the presence of redox probes, i.e., Fe $2+/ 3+$. The Fe $2+/ 3+$ couple displays a facile and non-catalytic electron transfer at the conducting electrodes, so the electrochemical measurement reveals the material properties encompassing the electrode conductivity and surface area without any reaction kinetic limitation. Figure 4a and $b$ are the Bode and Nyquist plots of the PPy/HA-modified electrodes. All the PPy/HAmodified electrodes displayed significantly lower impedance than unmodified bare ITO 
electrodes at frequencies lower than $103 \mathrm{~Hz}$. The impedance magnitudes of the PPy/HA significantly decreased as the HA MWs in the PPy/HA films increased. For example, total impedance magnitudes at $1 \mathrm{~Hz}$ were $970 \pm 216,750 \pm 125,680 \pm 30,580 \pm 80$, and $560 \pm 35$ Ohm for $\mathrm{PPy} / \mathrm{HA}_{35 \mathrm{k}}, \mathrm{PPy} / \mathrm{HA}_{100 \mathrm{k}}, \mathrm{PPy} / \mathrm{HA}_{500 \mathrm{k}}, \mathrm{PPy} / \mathrm{HA}_{1 \mathrm{M}}$, and $\mathrm{PPy} / \mathrm{HA}_{3 \mathrm{M}}$, respectively (Figure 4a and Supplementary Information Figure S3). Nyquist plots of the EIS spectra and their fitting with an equivalent circuit (Randles model with the Warburgh parameter) revealed the gradual decrease in charge transfer resistance (RCT) values with increases in HA MWs in PPy/HA (Figure 4b). RCT values of PPy/HA $35 \mathrm{k}$ and PPy/HA 3 m were $431 \pm 166$ and $170 \pm 16$ $\Omega$, respectively, showing a 2.5 fold difference (Figure $4 \mathrm{c}$ ). It should also be noted that no substantial trend was found for double-layer capacitance $(\mathrm{Cdl})$ for the PPy/HA films. Cyclic voltammetry showed the electrochemical activities of PPy/HA with distinct oxidation and reduction peaks in their cyclic voltammograms (Figure 4d). Charge storage capacitance (CSC) gradually increased with increases in the MWs of HA dopants in PPy/HA electrodes (Figure 4e). Higher CSC generally benefits charge injection at the interfaces of electrodes and medium and allows for efficient electrical signal mediation. Peak-to-peak separation voltages, indicating the conductance of the electrodes, were narrower in the cyclic voltamograms from the PPy/HA electrodes of higher MW HA dopants (Figure 4f). These results indicated that high MW HA dopants promote the electrical and electrochemical properties of PPy/HAmodified electrodes. This might be attributed to higher hydrophilicity leading to better wetting, the abundant negative charges aiding the formation of ordered structures during deposition, and the prevention of de-doping in the medium. 
a

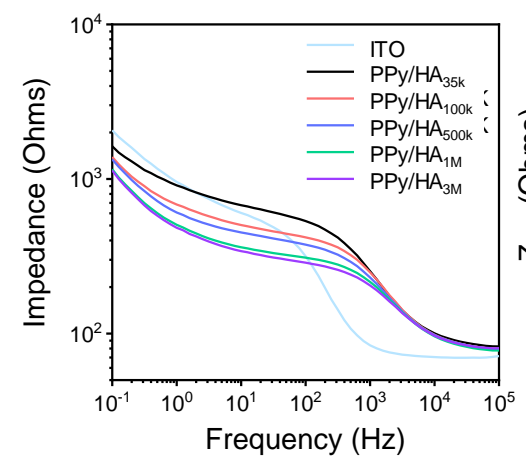

d

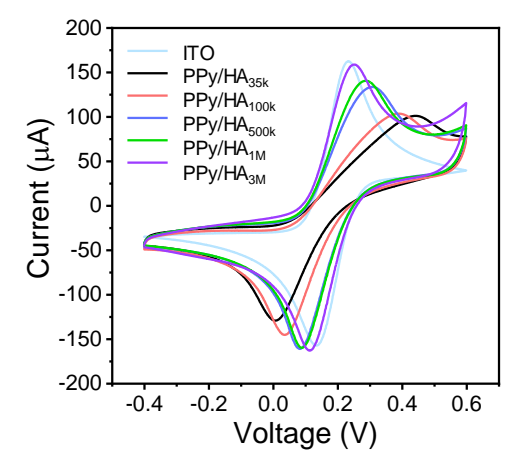

b



e

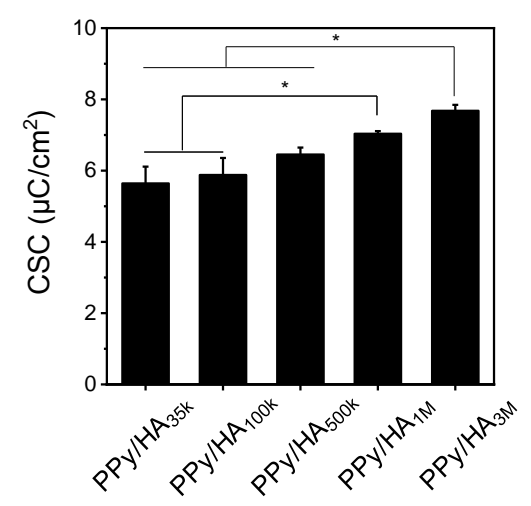

C


Figure 4. Electrochemical properties of PPy/HA-modified electrodes. (a) Bode and (b) Nyquist plots of impedance spectra of the various PPy/HA-modified electrodes. (c) Charge transfer resistances $\left(\mathrm{R}_{\mathrm{ct}}\right)$ calculated from the EIS spectra data by fitting with Randles circuit. Experiments were performed in PBS (pH 7.4) containing $5 \mathrm{mM} \mathrm{K}_{3} \mathrm{Fe}(\mathrm{CN})_{6}$. (d) Cyclic voltammograms of various PPy/HA-modified electrodes. $\mathrm{CV}$ was performed in $0.1 \mathrm{M} \mathrm{KCl}$ containing $5 \mathrm{mM} \mathrm{K}_{3} \mathrm{Fe}(\mathrm{CN})_{6}$ at $10 \mathrm{mV} / \mathrm{s}$ scan rates. (e) Charge storage capacitances and (f) peak-to-peak separation voltages.

\subsection{In vitro cell culture on the PPy/HA substrates}

To examine the cellular responses to MWs of HA dopants in PPy/HA, NIH3T3 fibroblasts and SH-SY5Y neuroblastoma were cultured on the different PPy/HA electrodes. Dramatic changes in cellular behaviors were observed depending on the MWs of HA dopants. (Figure 5) Both NIH3T3 and SH-SY5Y cells adhered and proliferated well on the PPy/HA $35 \mathrm{k}$, which were similar on bare ITO. Interestingly, very few cells were found on the high MW HA- 
doped PPy/HA, especially PPy/HA $1 \mathrm{M}$ and PPy/HA $3 \mathrm{M}$. These results indicate that the PPy/HA prepared with low MW HA $\left(<500 \times 10^{3} \mathrm{Da}\right)$ can support cell adhesion and growth, which would be beneficial for tissue scaffold applications. As the MWs of HA dopants increased, cell attachment and growth were dramatically decreased for both types of the tested cells, and few cells were found on the PPy/HA films prepared with $1 \times 10^{6}$ and $3 \times 10^{6}$ Da HA. Moreover, metabolic activities of the cells significantly increased during the incubation for the samples except PPy/HA $1 \mathrm{M}$ and $\mathrm{PPy} / \mathrm{HA}_{3 \mathrm{M}}$. These non-cell binding surfaces of conductive biomaterials would be beneficial to create non-fouling bioelectrodes considering the biofouling involving non-specific cell adhesion and scarring plays decisive roles in impairing electrode functions and tissue compatibility.[6,21,44] Conductive electrodes capable of minimizing non-specific cell adhesion can attenuate insulating cell layer formation and scarring around implanted bioelectrodes. As shown in Figure 5b, the metabolic activity of the cells (NIH3T3 and SH-SY5Y) on the PPy/HA $35 \mathrm{k}$ was as high as the cells on bare ITO. The metabolic activities gradually decreased with increases of HA MWs in the PPy/HA films and finally reached minimal values when the HA MWs were $\geq 500 \times 10^{5} \mathrm{Da}$. These results indicate that the MWs of HA dopant in PPy/HA films play decisive roles in modulating cellular behaviors. In general, low MW HA was reported to induce cell proliferation, while high MW HA possesses an anti-proliferative activity.[37] Furthermore, protein adsorption tests indicated that serum protein adsorption onto the PPy/HA samples decreased with an increase in molecular weight of HA in the samples (Supplementary Information Figure S4).[45] We believe that the highly hydrophilic nature of high MW HA doped-PPy/HA surfaces controls the non-cell adhesive properties by preventing protein adsorption and/or denaturation which subsequently leads to cell adhesion.[46,47] Note that previous studies with PPy/HA, carried out by other groups, reported generally good adhesion and growth on the PPy/HA substrates; however, these studies did not explore the effects of MWs of HA dopants in PPy/HA (and in certain cases the MW of the HA used was not reported). In this 
study, we successfully demonstrate that cell adhesion and proliferation on conductive PPy can be simply and effectively modulated by selecting the MW of HA used in the PPy/HA films.
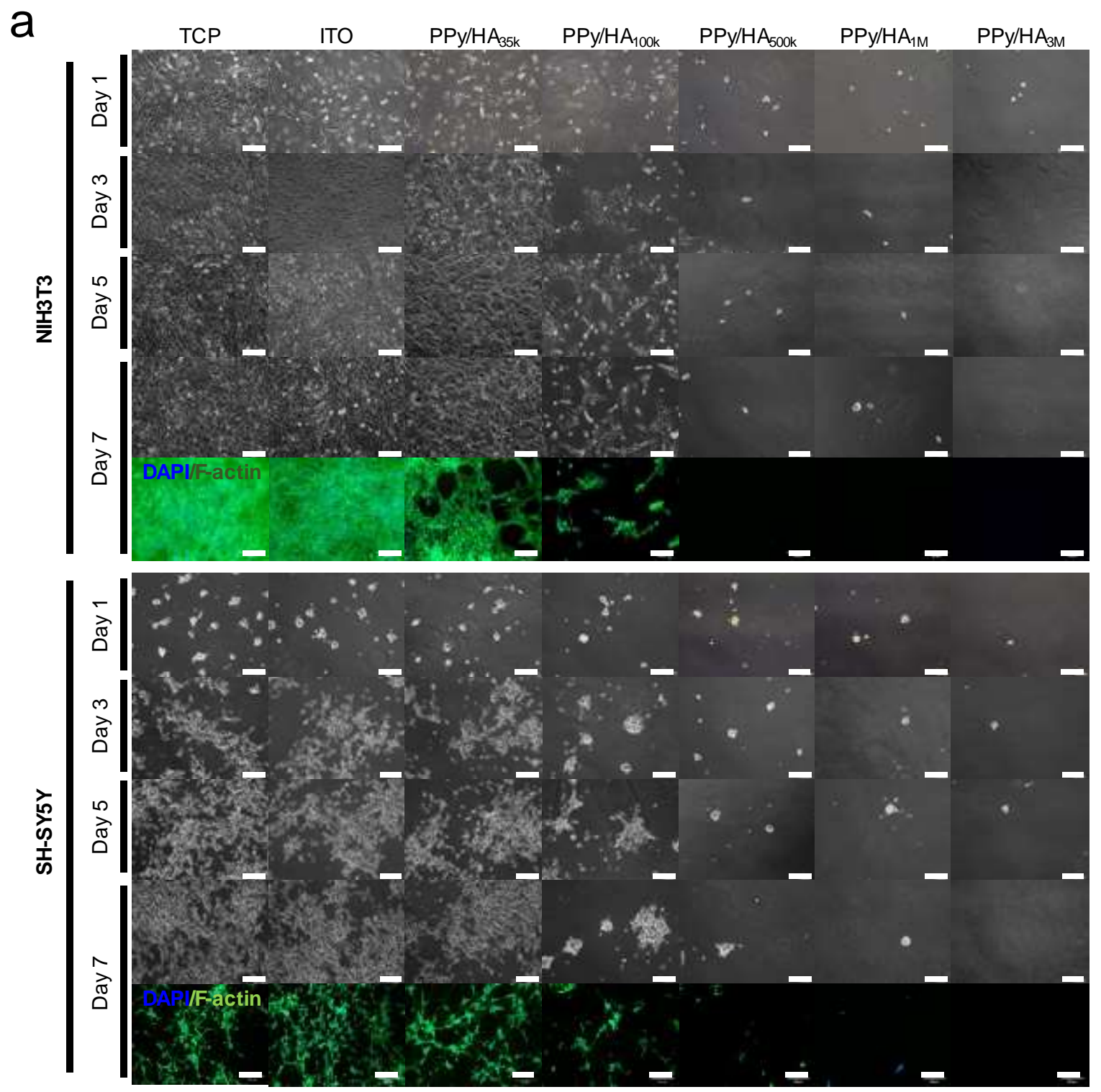

b


Figure 5. In vitro culture of NIH3T3 fibroblasts and SH-SY5Y neuroblastoma on the various PPy/HA substrates. (a \& b) Optical and immunofluorescence images of NIH3T3 cells and 
SH-SY5Y cells. Scale bars represent $200 \mu \mathrm{m}$. At day 7, the cells were stained for $F$-actin (green) and nuclei (blue) using Alexa488-conjugated phalloidin and DAPI, respectively. Metabolic activities of the cells during 7 day culture on the PPy/HA films. Statistical significance was evaluated using two-way ANOVA with Bonferroni's post-test. $* p<0.05$ indicates significant different between samples. Hash symbol denotes significance $(p<0.05)$ relative to the Day1 samples.

\subsection{In vivo tissue compatibility and EMG signal recording}

The tissue compatibility of the PPy/HA was assessed by subcutaneous implantation of the samples for 4 weeks (Figure 6a). The tissues were analyzed by Masson Trichrome staining. During the implantation, no severe symptoms of tissue inflammation, such as redness or swelling, was observed for all samples. Tissues implanted with PPy/HA substrates exhibited significantly lower scar tissue formation compared to unmodified bare substrates (Figure 6b). However, no difference in scar tissue thickness was observed among different PPy/HA, confirming the modification of the ITO resulted in improved tissue. HA, especially high MW HA, is known to exhibit an anti-inflammatory activity. Rayahin et al. demonstrated that inflammatory macrophage activation could be suppressed by high MW HA $\left(3 \times 10^{6} \mathrm{Da}\right)$.[42] Also, Khaing et al. reported the attenuation of scar tissue formation post spinal cord injuries by providing high MW HA.[48] We observed reduced scar tissue formation even from low MW $\left(35 \times 10^{3} \mathrm{Da}\right)$ HA-doped PPy/HA. Therefore, PPy/HA films prepared with a large range of HA MWs $\left(35 \times 10^{3}-3 \times 10^{6} \mathrm{Da}\right)$ should be suitable as biocompatible implant materials. As a proof-of-concept, we utilized PPy/HA-modified electrodes for in vivo EMG signal measurement. The electrodes were implanted into sciatic nerves and measured the evoked EMG signals from the tibia muscle while stimulating the sciatic nerve (Figure 6c). PPy/HAmodified electrodes permitted more sensitive signal recording compared to unmodified

Figure 6. In vitro culture of NIH3T3 fibroblasts and SH-SY5Y neuroblstoma on the various PPy/HA substrates. Optical and immunofluorescence images of (a) NIH3T3 cells and (b) SH-SY5Y cells. Scale bars represent 200 $\mu \mathrm{m}$. At day 7 , the cells were stained for $\boldsymbol{F}$-actin (green) and nuclei (blue) using Alexa488-conjugated phalloidin and DAPI, respectively. (c) Metabolic activities of the cells during 7 day culture on the PPy/HA films. Statistical significance was evaluated using one-way ANOVA with $p=0.05$. 
electrodes (Figure 6d). In addition, PPy/HA3M-modified electrodes exhibited higher sensitivity in EMG signal detection than PPy/HA35k-modified electrodes as evident by larger peak-to-peak amplitude and high signal-to-noise ratio (Figure 6e, f). The improved signal sensitivity from PPy/HA-modified electrodes can be attributed to their improved electrochemical properties, such as high CSC and low impedances (low RCT). Also, higher surface area and hydrophilicity would aid highly sensitive detection of in vivo electrical signals from high MW HA-doped PPy/HA.

a
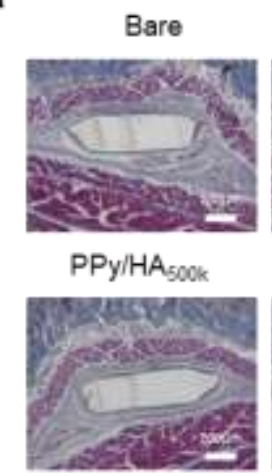

C


b


f

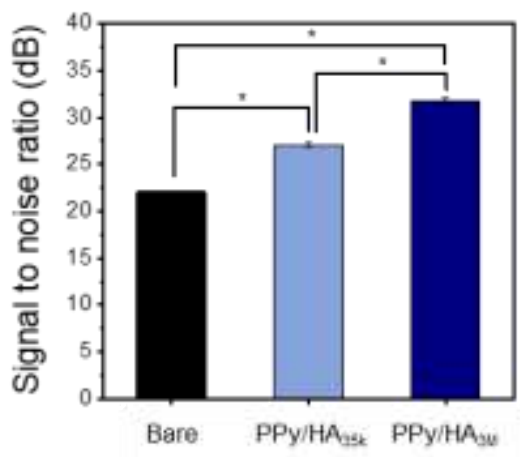


Figure 6. In vivo tests for tissue compatibility and EMG signal recording. (a) Masson Trichrome staining images of the tissues implanted bare and PPy/HA-modified electrodes. Substrates were subcutaneously implanted for 4 weeks. Scale bars represent $200 \mu \mathrm{m}$. (b) Scar tissue thickness in the tissues implanted with various PPy/HA substrates. (c) A schematic illustration of the electrophysiological EMG signal measurement at tibialis anterior using an implantable concentric needle electrode by stimulating sciatic nerve using a hook-type electrode. (d) In vivo EMG signals obtained from bare, $\mathrm{PPy} / \mathrm{HA}_{35 \mathrm{k}}$, and $\mathrm{PPy} / \mathrm{HA}_{3 \mathrm{M}}$ electrodes. (e) Average peak-to-peak amplitudes and (f) signal-to-noise ratios of the obtained EMG signals. A concentric needle electrode, either modified or modified with PPy/HA, was implanted to rat tibia and measured EMG signals while stimulating sciatic nerve with a hook electrode with $0.7 \mathrm{~V}$.

\section{Conclusions}

Biomimetic CP-based materials have shown their utilities to endow specific biological abilities as well as electrical/electrochemical activities. In this present study, various HAincorporated PPy were electrochemically polymerized by differing MWs of HA dopants. Careful investigation of the material characteristics of these PPy/HA revealed pivotal roles of HA MWs in their electrochemical and biological properties. Small MW HA-doped PPy well supported adhesion and proliferation of fibroblasts and neuronal cells, while high MW HAdoped PPy exhibited highly hydrophilic surfaces that resist cell growth. The results clearly demonstrate the simple and effective engineering of PPy/HA electrodes' properties by simply control over MW of HA. Furthermore, we successfully demonstrated improved tissue compatibility and the performances of in vivo EMG signal acquisition of the PPy/HA. In conclusion, biomimetic PPy/HA-based biomaterials can serve as versatile platforms that can exhibit dramatically different characters by controlling the MWs of HA dopants. Our findings 
and demonstration will greatly benefit the development of various conductive biomaterials, such as implantable electrodes, biosensors, and scaffolds for tissue engineering.

\section{Declaration of interest}

There are no conflicts of interest to declare. All authors have given approval to the final version of the manuscript.

\section{Author Contributions}

S.K., Y.J. and J.L. designed the study; S.K. and Y.J. performed the study; M.J. performed synthesis of the samples; A.L. performed AFM, KFPM analysis; H.P. analyzed electrochemical data; all au-thors participated in development and discussions; $\$$ These authors contributed equally.

\section{Acknowledgment}

This research was supported by the Basic Science Research Program (2016R1A2B4007172), Pioneer Research Center Program (2014M3C1A3001208), Global Research Laboratory (GRL) Program (2013K1A1A2A02050616) through the National Research Foundation of Korea (NRF) funded by the Ministry of Science, ICT \& Future Planning. This research was also supported by a grant of the Korea Health Technology R\&D Project through the Korea Health Industry Development Institute (KHIDI), funded by the Ministry of Health \& Welfare (HI14C3484). JGH acknowledges the support of a Lancaster University Faculty of Science and Technology Early Career Internal Grant and a Royal Society Research Grant (RG160449).

\section{References}


[1] R. Balint, N.J. Cassidy, S.H. Cartmell, Conductive polymers: towards a smart biomaterial for tissue engineering, Acta Biomater. 10 (2014) 2341-2353.

[2] G.G. Wallace, S.E. Moulton, G.M. Clark, Electrode-cellular interface, Sci. Publ. Vol 14 2004-2012. (2009).

[3] Z. Shi, X. Gao, M.W. Ullah, S. Li, Q. Wang, G. Yang, Electroconductive natural polymer-based hydrogels, Biomaterials. 111 (2016) 40-54.

[4] Y. Wu, Y.X. Chen, J. Yan, D. Quinn, P. Dong, S.W. Sawyer, P. Soman, Fabrication of conductive gelatin methacrylate-polyaniline hydrogels, Acta Biomater. 33 (2016) 122130.

[5] C. Xie, P. Li, L. Han, Z. Wang, T. Zhou, W. Deng, K. Wang, X. Lu, Electroresponsive and cell-affinitive polydopamine/polypyrrole composite microcapsules with a dualfunction of on-demand drug delivery and cell stimulation for electrical therapy, NPG Asia Mater. 9 (2017) e358.

[6] B. Zhu, S.-C. Luo, H. Zhao, H.-A. Lin, J. Sekine, A. Nakao, C. Chen, Y. Yamashita, H. $\mathrm{Yu}$, Large enhancement in neurite outgrowth on a cell membrane-mimicking conducting polymer, Nat. Commun. 5 (2014) 4523.

[7] D. Khodagholy, T. Doublet, M. Gurfinkel, P. Quilichini, E. Ismailova, P. Leleux, T. Herve, S. Sanaur, C. Bernard, G.G. Malliaras, Highly conformable conducting polymer electrodes for in vivo recordings, Adv. Mater. 23 (2011).

[8] T.D.Y. Kozai, N.B. Langhals, P.R. Patel, X. Deng, H. Zhang, K.L. Smith, J. Lahann, N.A. Kotov, D.R. Kipke, Ultrasmall implantable composite microelectrodes with bioactive surfaces for chronic neural interfaces, Nat. Mater. 11 (2012) 1065.

[9] D. Khodagholy, T. Doublet, P. Quilichini, M. Gurfinkel, P. Leleux, A. Ghestem, E. Ismailova, T. Hervé, S. Sanaur, C. Bernard, In vivo recordings of brain activity using organic transistors, Nat. Commun. 4 (2013) 1575.

[10] D. Khodagholy, J.N. Gelinas, T. Thesen, W. Doyle, O. Devinsky, G.G. Malliaras, G. Buzsáki, NeuroGrid: recording action potentials from the surface of the brain, Nat. Neurosci. 18 (2015) 310.

[11] T. Someya, Z. Bao, G.G. Malliaras, The rise of plastic bioelectronics, Nature. 540 (2016) 379.

[12] E. Stewart, N.R. Kobayashi, M.J. Higgins, A.F. Quigley, S. Jamali, S.E. Moulton, R.M. Kapsa, G.G. Wallace, J.M. Crook, Electrical stimulation using conductive polymer polypyrrole promotes differentiation of human neural stem cells: a biocompatible platform for translational neural tissue engineering, Tissue Eng. Part C Methods. 21 (2014) 385-393.

[13] B. Weng, J. Diao, Q. Xu, Y. Liu, C. Li, A. Ding, J. Chen, Bio- Interface of Conducting Polymer- Based Materials for Neuroregeneration, Adv. Mater. Interfaces. 2 (2015).

[14] D. Ho, J. Zou, X. Chen, A. Munshi, N.M. Smith, V. Agarwal, S.I. Hodgetts, G.W. Plant, A.J. Bakker, A.R. Harvey, Hierarchical patterning of multifunctional conducting polymer nanoparticles as a bionic platform for topographic contact guidance, Acs Nano. 9 (2015) 1767-1774.

[15] B.S. Spearman, A.J. Hodge, J.L. Porter, J.G. Hardy, Z.D. Davis, T. Xu, X. Zhang, C.E. Schmidt, M.C. Hamilton, E.A. Lipke, Conductive interpenetrating networks of polypyrrole and polycaprolactone encourage electrophysiological development of cardiac cells, Acta Biomater. 28 (2015) 109-120.

[16] M.R. Aufan, Y. Sumi, S. Kim, J.Y. Lee, Facile synthesis of conductive polypyrrole wrinkle topographies on polydimethylsiloxane via a swelling-deswelling process and their potential uses in tissue engineering, ACS Appl. Mater. Interfaces. 7 (2015) 2345423463. 
[17] K. Nagamine, H. Sato, H. Kai, H. Kaji, M. Kanzaki, M. Nishizawa, Contractile Skeletal Muscle Cells Cultured with a Conducting Soft Wire for Effective, Selective Stimulation, Sci. Rep. 8 (2018) 2253.

[18] C. Kleber, M. Bruns, K. Lienkamp, J. Rühe, M. Asplund, An interpenetrating, microstructurable and covalently attached conducting polymer hydrogel for neural interfaces, Acta Biomater. 58 (2017) 365-375.

[19] R. Bao, B. Tan, S. Liang, N. Zhang, W. Wang, W. Liu, A $\pi-\pi$ conjugation-containing soft and conductive injectable polymer hydrogel highly efficiently rebuilds cardiac function after myocardial infarction, Biomaterials. 122 (2017) 63-71.

[20] S. Yang, L. Jang, S. Kim, J. Yang, K. Yang, S. Cho, J.Y. Lee, Polypyrrole/alginate hybrid hydrogels: electrically conductive and soft biomaterials for human mesenchymal stem cell culture and potential neural tissue engineering applications, Macromol. Biosci. 16 (2016) 1653-1661.

[21] W. Wang, M. Cui, Z. Song, X. Luo, An antifouling electrochemical immunosensor for carcinoembryonic antigen based on hyaluronic acid doped conducting polymer PEDOT, RSC Adv. 6 (2016) 88411-88416.

[22] G. Wang, X. Su, Q. Xu, G. Xu, J. Lin, X. Luo, Antifouling aptasensor for the detection of adenosine triphosphate in biological media based on mixed self-assembled aptamer and zwitterionic peptide, Biosens. Bioelectron. 101 (2018) 129-134.

[23] J. Wang, M. Jiang, Toward genolelectronics: Nucleic acid doped conducting polymers, Langmuir. 16 (2000) 2269-2274.

[24] J.D. Nickels, C.E. Schmidt, Surface modification of the conducting polymer, polypyrrole, via affinity peptide, J. Biomed. Mater. Res. A. 101 (2013) 1464-1471.

[25] Y. Li, C. Yu, RGD peptide doped polypyrrole film as a biomimetic electrode coating for impedimetric sensing of cell proliferation and cytotoxicity, J. Appl. Biomed. 15 (2017) 256-264.

[26] M.D. Levi, C. Lopez, E. Vieil, M.A. Vorotyntsev, Influence of ionic size on the mechanism of electrochemical doping of polypyrrole films studied by cyclic voltammetry, Electrochimica Acta. 42 (1997) 757-769.

[27] R.A. Green, N.H. Lovell, L.A. Poole-Warren, Impact of co-incorporating laminin peptide dopants and neurotrophic growth factors on conducting polymer properties, Acta Biomater. 6 (2010) 63-71.

[28] S. Baek, R.A. Green, L.A. Poole- Warren, Effects of dopants on the biomechanical properties of conducting polymer films on platinum electrodes, J. Biomed. Mater. Res. A. 102 (2014) 2743-2754.

[29] B.C. Thompson, S.E. Moulton, R.T. Richardson, G.G. Wallace, Effect of the dopant anion in polypyrrole on nerve growth and release of a neurotrophic protein, Biomaterials. 32 (2011) 3822-3831.

[30] J.A. Burdick, G.D. Prestwich, Hyaluronic acid hydrogels for biomedical applications, Adv. Mater. 23 (2011).

[31] J.H. Collier, J.P. Camp, T.W. Hudson, C.E. Schmidt, Synthesis and characterization of polypyrrole-hyaluronic acid composite biomaterials for tissue engineering applications, J. Biomed. Mater. Res. 50 (2000) 574-584.

[32] K.J. Gilmore, M. Kita, Y. Han, A. Gelmi, M.J. Higgins, S.E. Moulton, G.M. Clark, R. Kapsa, G.G. Wallace, Skeletal muscle cell proliferation and differentiation on polypyrrole substrates doped with extracellular matrix components, Biomaterials. 30 (2009) 5292-5304.

[33] Y. Kobayashi, A. Okamoto, K. Nishinari, Viscoelasticity of hyaluronic acid with different molecular weights, Biorheology. 31 (1994) 235-244.

[34] R. Stern, A.A. Asari, K.N. Sugahara, Hyaluronan fragments: an information-rich system, Eur. J. Cell Biol. 85 (2006) 699-715. 
[35] R. Prevo, S. Banerji, D.J. Ferguson, S. Clasper, D.G. Jackson, Mouse LYVE-1 is an endocytic receptor for hyaluronan in lymphatic endothelium, J. Biol. Chem. 276 (2001) 19420-19430.

[36] J.M. Cyphert, C.S. Trempus, S. Garantziotis, Size matters: molecular weight specificity of hyaluronan effects in cell biology, Int. J. Cell Biol. 2015 (2015).

[37] J. Li, F. Wu, K. Zhang, Z. He, D. Zou, X. Luo, Y. Fan, P. Yang, A. Zhao, N. Huang, Controlling Molecular Weight of Hyaluronic Acid Conjugated on Amine-rich Surface: Toward Better Multifunctional Biomaterials for Cardiovascular Implants, ACS Appl. Mater. Interfaces. 9 (2017) 30343-30358.

[38] J.E. Rayahin, J.S. Buhrman, Y. Zhang, T.J. Koh, R.A. Gemeinhart, High and low molecular weight hyaluronic acid differentially influence macrophage activation, ACS Biomater. Sci. Eng. 1 (2015) 481-493.

[39] H. Saari, Y.T. Konttinen, C. Friman, T. Sorsa, Differential effects of reactive oxygen species on native synovial fluid and purified human umbilical cord hyaluronate, Inflammation. 17 (1993) 403-415.

[40] N. Gomez, C.E. Schmidt, Nerve growth factor- immobilized polypyrrole: Bioactive electrically conducting polymer for enhanced neurite extension, J. Biomed. Mater. Res. A. 81 (2007) 135-149.

[41] B. Liu, X. Liu, S. Shi, R. Huang, R. Su, W. Qi, Z. He, Design and mechanisms of antifouling materials for surface plasmon resonance sensors, Acta Biomater. 40 (2016) $100-118$.

[42] M.L. Carman, T.G. Estes, A.W. Feinberg, J.F. Schumacher, W. Wilkerson, L.H. Wilson, M.E. Callow, J.A. Callow, A.B. Brennan, Engineered antifouling microtopographiescorrelating wettability with cell attachment, Biofouling. 22 (2006) 11-21.

[43] J.Y. Lee, C.E. Schmidt, Pyrrole-hyaluronic acid conjugates for decreasing cell binding to metals and conducting polymers, Acta Biomater. 6 (2010) 4396-4404.

[44] S. Kim, Y. Jang, L.K. Jang, S.H. Sunwoo, T. Kim, S.-W. Cho, J.Y. Lee, Electrochemical deposition of dopamine-hyaluronic acid conjugates for anti-biofouling bioelectrodes, $\mathrm{J}$. Mater. Chem. B. 5 (2017) 4507-4513.

[45] L. Li, M. Yu, P.X. Ma, B. Guo, Electroactive degradable copolymers enhancing osteogenic differentiation from bone marrow derived mesenchymal stem cells, J. Mater. Chem. B. 4 (2016) 471-481.

[46] S. Chen, L. Li, C. Zhao, J. Zheng, Surface hydration: principles and applications toward low-fouling/nonfouling biomaterials, Polymer. 51 (2010) 5283-5293.

[47] Y. Qian, L. Li, Y. Song, L. Dong, P. Chen, X. Li, K. Cai, O. Germershaus, L. Yang, Y. Fan, Surface modification of nanofibrous matrices via layer-by-layer functionalized silk assembly for mitigating the foreign body reaction, Biomaterials. 164 (2018) 22-37.

[48] Z.Z. Khaing, B.D. Milman, J.E. Vanscoy, S.K. Seidlits, R.J. Grill, C.E. Schmidt, High molecular weight hyaluronic acid limits astrocyte activation and scar formation after spinal cord injury, J. Neural Eng. 8 (2011) 046033. 